\title{
Enhanced Cluster Stability in Vehicular ad hoc Network
}

\author{
K. V. Rop ${ }^{1, *}$, P. K. Langat ${ }^{2}$, H. A. Ouma ${ }^{3}$ \\ ${ }^{1}$ Department of Electrical Engineering, Pan African University, Institute for Basic Sciences, Technology \& Innovation, Kenya \\ ${ }^{2}$ Department of Telecommunication and Information Engineering, Jomo Kenyatta University of Agriculture \&Technology, Kenya \\ ${ }^{3}$ Department of Electrical and Information Engineering, University of Nairobi, Kenya
}

Copyright $\odot 2019$ by authors, all rights reserved. Authors agree that this article remains permanently open access under the terms of the Creative Commons Attribution License 4.0 International License

\begin{abstract}
Traffic management has been and remains to be a major problem especially in urban areas with high vehicle density. Adoption of intelligent transport system (ITS) has been widely experimented with the intention of curbing traffic menace with mixed experiences as the outcome. By communicating with other vehicles travelling on the same road in form of clusters, vehicular ad hoc network (VANET) forms an ITS that can allow cooperation of vehicles with less human input. By considering the high mobility nature of the vehicles in VANET, this paper presents a solution to the main menace of VANET clustering by adopting the flexibility of fuzzy logics for cluster formation on a multilane urban highway. It also shows that the stability of clusters is improved by performing the cluster head $(\mathrm{CH})$ selection process based on a combination of fuzzy logics, lane weighting, and utility function with the fuzzy membership function adjusted to increase stability of clusters.
\end{abstract}

Keywords Clustering, Vehicular ad hoc Network, Fuzzy Logic, Lane Weighting

\section{Introduction}

Vehicles in a route travelling in one direction have similar characteristics related to direction of travel, relative speeds, and proximity to each other. These vehicles can be grouped in a manner that allows effective and efficient management and sharing of information with each other. Vehicular ad hoc network (VANET) is an example of an intelligent transport system (ITS) with self-organizing network architecture that comprises of vehicles (such as motorcars, motorcycles, and buses), commonly referred to as nodes, travelling in the same direction and within a communication radius [1]-[3]. In VANET vehicles within a communication range can exchange various information such as road safety messages (e.g a stationary vehicle or accident warning) and commercial application messages (e.g. video and audio streaming) [4], [5].
Vehicle in a VANET contains an On-Board Unit (OBU) which is an embedded module that allows vehicle-to-vehicle (V2V), vehicle-to-person (V2P), or vehicle-to-infrastructure (V2I), communication [2], [6]. The most common arrangement allows V2V or V2I communication where the infrastructure in V2I is the roadside unit (RSU) which is stationery. Since VANET topology is not random but dynamic and predictable, it is possible for vehicles to communicate and share information for an extended period of time without breaking the link. This communication is made possible by utilization of Dedicated Short-Range Communication (DSRC), designed with $75 \mathrm{MHz}$ band between 5.850 and $5.925 \mathrm{GHz}$ of the frequency spectrum based on IEEE 802.11p and IEEE 1609.X standard for supporting various vehicular communication applications [7], [8].

Due to high mobility nature of the nodes in a VANET, network topology may change often causing instability in the network as the network may not be robust and adaptive enough [9]. Also, with the limited bandwidth of $75 \mathrm{MHz}$ allocated for communication, breakdown in communication links are bound to be experienced. The solution to this problem is the application of clustering techniques which partition the network into smaller units. This partitioning process which is done based on some clustering algorithm will group the nodes in a manner that ensures longer cluster life thus reducing the reclustering process. A cluster consists of nodes within given regional boundaries that have been grouped together and are able to communicate with each other. These nodes in a cluster can be categorized as cluster members (CM) who are the communicating nodes in a cluster or cluster head $(\mathrm{CH})$ who coordinates the activities of a cluster. This distinct classification of $\mathrm{CM}$ and $\mathrm{CH}$ provides a structure way of communicating fairly [1], [5], [7], [10].

The main challenge with clustering system is instability due to vehicle mobility. Most of the researchers have proposed different complex techniques and algorithms for clustering [2], [11]-[13], however, they fail to consider that the best systems are the simplest systems. Artificial intelligence provides unique feature of optimization that 
can incorporate different methodologies to achieve an optimized output [14]-[17]. In this paper, we propose a clustering technique based on fuzzy logic which based on human reasoning. The proposed novel stability fuzzy logic based clustering algorithm (SFCA) reduces the reclustering overhead while providing an efficient network topology which is hierarchical. The primary focus in this paper is the stability of clusters and effective communication within a cluster. The novel contributions here include;

- Adaptive and simplified stable cluster head selection methodology achieved by means of fuzzy reasoning that can be applied both for one way or two ways multilane or single lane highways.

- Incorporation of lane weighting in $\mathrm{CH}$ selection to provide longer lifeline of a $\mathrm{CH}$. This gives preference to the node at the middle lanes since the probability of exiting at the next junction is lower.

The organization of the rest of the paper is as follows. Section II discusses briefly related works that have been published on this area. Section III discusses the novel clustering and $\mathrm{CH}$ selection process elaborating on the parameters to be considered during this process. Section IV and $\mathrm{V}$ discusses the simulation results and concludes the work respectively.

\section{Related Work}

The last few years has experienced a growth in ITS interest and development with the main focus on how vehicles in a network can effectively and efficiently share information with one another. Previous researches have shown that depending on the mobility metrics adopted, clustering of vehicles in VANET achieve higher throughput as compared to non-clustered vehicles. Some of the latest clustering techniques are presented in this section, without purporting to exhaustively cover the VANET field.

A clustering algorithm based on the lowest-ID algorithm called mobility-based clustering algorithm (MOBIC) is proposed in [18]. MOBIC has its basis on the selection of the $\mathrm{CH}$ with the lowest ID, with each node having a fixed ID. In MOBIC, nodes broadcast their IDs to other vehicular nodes in range. Each node then determines the $\mathrm{CH}$ as the node with lowest ID. MOBIC is a very simple and basic algorithm that is meant for mobile ad hoc networks (MANETs) and does not work well in VANETs; nevertheless, it forms a basis for comparison purposes. An algorithm called affinity propagation (AP) is one of the recently proposed stable algorithms for cluster formation [19]. Cluster stability is remarkably improved by application of AP to VANETs. Since AP is a distance-based algorithm, frequent change of $\mathrm{CHs}$ are common with dramatic change is speed of the vehicle. Moreover, AP has higher cluster formation time delay due to several iterative loops required. Also, one major shortcoming of this algorithm in lack of consideration of neighbors' vehicular speed differences.

Souza et al. [20] proposed an Aggregate Local Mobility (ALM) based clustering technique. In ALM, the sender and receiver distance difference is calculated by using the Received Signal Strength (RSS). The relative mobility between the two nodes is taken from the ratio of the RSS in disseminated two successive hello messages. However, in this system, clear signals are hard to come by because of noise accompanying the signals. For improved cluster lifetime stability in VANETs, a lane-based clustering algorithm is proposed in [21]. The author uses urban scenario for illustration in this in work. The authors argue that for more cluster stability, fewer $\mathrm{CH}$ changes are required. It is paramount for a node with the potential of being the $\mathrm{CH}$ for the longest time in this network to be selected as the $\mathrm{CH}$. [22] proposes a VANET algorithm based on hybrid backbone algorithm. The authors in this paper proposed an initial formation of a leadership designated backbone by the nodes sharing higher degree of connectivity in the network. Using aggregate relative velocity of the nodes in the leadership, a selection of $\mathrm{CH}$ in initiated. Also, re-organization of the cartels is handled through this leadership. However, this methodology slows down the formation of $\mathrm{CH}$ in a non-delay tolerant network. The Utility Function algorithm [23], an algorithm uses velocity, distance, and connectivity level for calculating node weights. The node with the most average of the three above mentioned parameters is selected as a $\mathrm{CH}$. Also, all the nodes must periodically broadcast their status to other nodes in the neighborhood and within range. The proposed AMACAD [24] which is based on the final vehicle's destination rallies on the concept that there is a high probability of vehicles heading to the same destination to retain the same cluster. Therefore, relative and final destination, relative speed, and present position are considered in cluster formation.

The proposed Intelligent Based Clustering Algorithm in VANET (IBCAV) [25] uses a combination of artificial neural network (ANN) and training its network using genetic algorithm. This method however gives preference to a vehicle with lower speed than the rest which may cause frequent reclustering if the other neighboring vehicles are moving at a relatively higher speed. In [3], a NNPAC (Neural Network based Partitioning around Cluster head) is proposed which tries to select the $\mathrm{CH}$ based on parameters under different scenarios. The authors argues that $\mathrm{CH}$ selection in most algorithms purely totally depend on the static parameter values. Apart from not considering the bias value in application of ANN, the research focused on single hop neighbors which can lead to formation of too many clusters in a high vehicular density area. A fuzzy logic based clustering methodology that uses the direction of movement, acceleration, and speed is proposed in [26]. A highway with two-way multilane is considered in [13] while [24] uses $\mathrm{CH}$ 
stability criterion where a certain threshold must be maintained for a vehicle to remain as a $\mathrm{CH}$. Calhan [15] proposes a fuzzy logic based cluster head selection algorithm (CHSA) with the fuzzy logic input parameters as the speed, distance, acceleration, and direction. The authors argue that the proposed CHSA fuzzy logic performs in both low speed and high speed vehicles environment on two-way multilane highway. In [17], the authors propose a rough set based fuzzy clustering in formation of clusters in a VANET. Here, the authors argue that rough set based fuzzy scheme when compared to other existing clustering approaches achieves $10 \%$ to $20 \%$ more average cluster lifetime and $20 \%$ to $25 \%$ more cluster head lifetime. The authors however, fail to clearly state the nature of the road network used (whether a single lane or two-way multilane highway). Also, speed, location, and direction were used as the only input parameters into the fuzzy system, thus fails to consider the distance vector.

In this work, a novel stability fuzzy logic based clustering algorithm (SFCA) is proposed that takes the advantage of logical reasoning when selecting the $\mathrm{CH}$. It combines both the utility function and lane based clustering to produce an effective stable clustering algorithm for use in VANETs.

\section{VANET Clustering Process}

\subsection{Network Model}

The proposed network model is based on a two-way high vehicular density highway. Vehicles on the same direction are clustered into one cluster depending on the geographical boundaries and connectivity levels. At no time can vehicles heading on opposite direction be clustered together. RSU are used to aid in initialization of the clustering process and at the onset of clustering, every $\mathrm{CM}$ has an equal chance of being selected as the $\mathrm{CH}$. Figure 1 shows a simple illustration of a road network with linkages shown. The following procedures are essential during clustering process.

i. Neighborhood Discovery: The first step in clustering process is discovery of neighboring nodes. A node on joining a road network first broadcasts a HELLO message with contains its information, while at the same time collecting its n-hop neighbors' information. This information will be kept in a neighbor table to be used for clustering.

ii. CH Selection: The next step is the initialization of $\mathrm{CH}$ selectin process. Each node's Clusterhead Level (CHL) is evaluated and broadcasted. This is done based on fuzzy logic and the node with the highest CHL weight becomes the $\mathrm{CH}$ by selection. This $\mathrm{CH}$ will perform various roles routing and linking with other $\mathrm{CH}$ in different clusters. Also, $\mathrm{CH}$ is responsible for inviting other cluster members to join in the cluster once they are within its range. If there in only one node in the area, it automatically becomes the $\mathrm{CH}$ and broadcast and invite for any node within that cluster radius to join.

iii. Affiliation and Invite: A $\mathrm{CH}$ broadcast invite message for all the nodes within the vicinity to join and participates in the cluster. The rest of the members on joining have become clusters members.

iv. Maintenance: Periodically, all members evaluate their link to its cluster $\mathrm{CH}$ to ensure that there is continuous link. Also, it is done periodically to confirm its status in terms of cluster affiliation. When the $\mathrm{CMs}$ fail to receive communication from $\mathrm{CH}$, reclustering process shall be initiated that will take us back to step 2 above. 


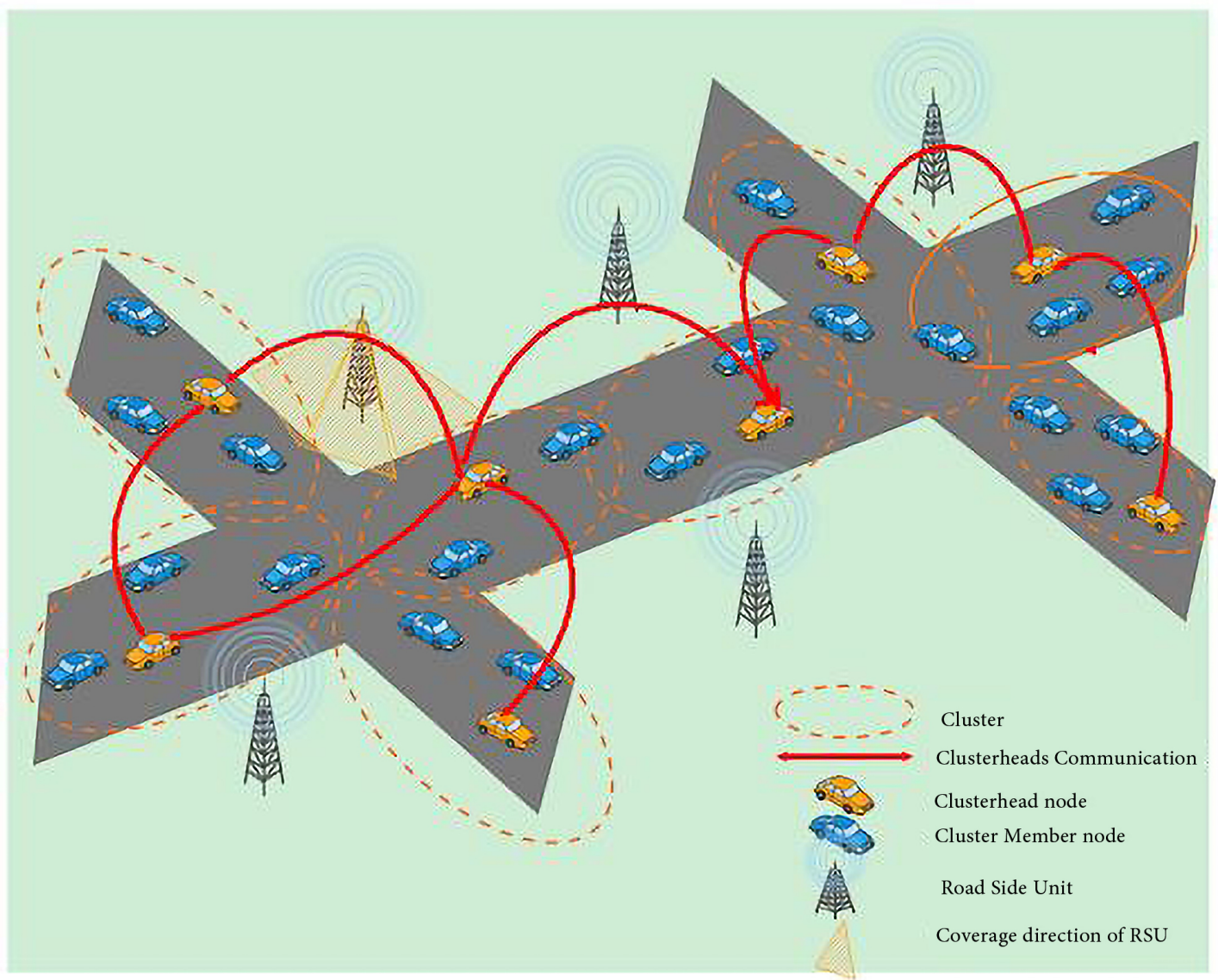

Figure 1. Illustration of a VANET Road Network

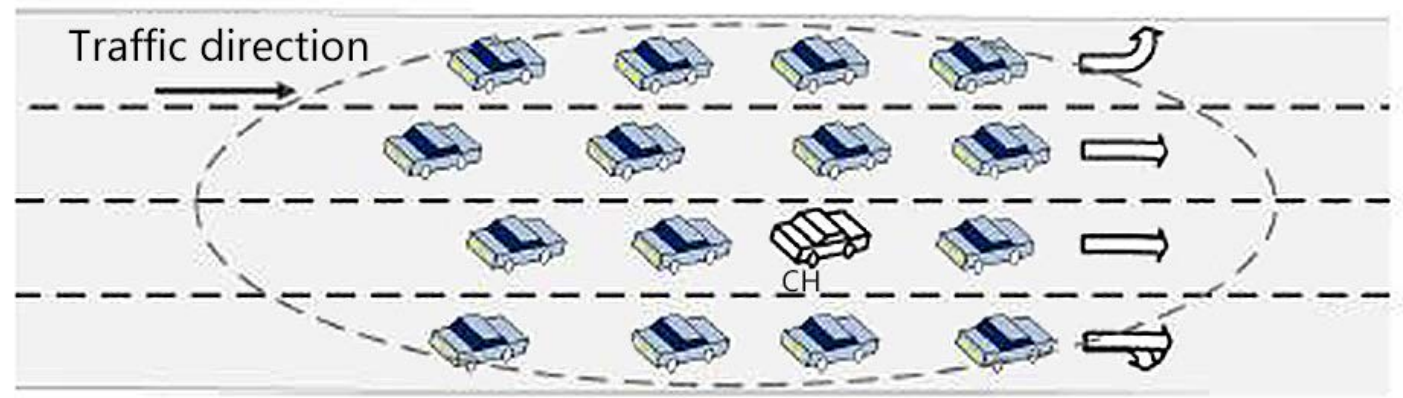

Figure 2. Lane Weighting Scenario

\subsection{CH Selection Process}

Cluster stability is a top priority when considering the criterion of a $\mathrm{CH}$ selection. A $\mathrm{CH}$ selection based on fuzzy logic methodology is considered where the parameters like speed, direction, lane weighting, and connectivity levels are used. These parameters are discussed below;

\subsubsection{Lane Weight}

Vehicular traffic in urban environment splits at each intersection into three as;
- $\quad$ Left Turn (LT) - This is where the traffic on the left most lane takes the left turn.

- Right Turn (RT) - This is where the traffic on the right most lane takes the right turn.

- No Turn (NT) - The traffic in this lane, mostly in the middle does not take a turn but goes straight.

The lane weighting aids in determining the vehicle's lane of travel. Using the number of lanes of each traffic flow (NLTF) and the total number of lanes on the road (TNL), the general lane weighing (LW) formula [21], is given as; 


$$
L W_{k}=\frac{1}{T N L} \times N L T F_{k}
$$

where, $\mathrm{k}$ is the vehicle lane number.

In this work, it is proposed that the $\mathrm{CH}$ should be a node that is in the middle lane as much as possible. This necessitates the varying of lane weight based on the lane the vehicle is at. To achieve this, it is proposed that the middle lane is allocated half the total weights while outer lanes share equally the remaining half. Eq. 1 can therefore be written as;

$$
L W_{k}=\left\{\begin{array}{l}
\frac{1}{T N L} \times N L T F_{k}, \text { for } T N L \leq 2 \\
\frac{2}{T N L} \times N L T F_{k}, \text { for } T N L>2 \\
\frac{1}{2 T N L} \times N L T F_{k}, \text { for } T N L>2
\end{array}\right.
$$

where, $\quad T F=N T$ for $T N L>2$ and $T F=R T=$ $L T$ for $T N L>2$

For example, if we have a road of three lanes with one LT, RT, and NT, then the LW for each traffic flow will be;

$$
\begin{gathered}
L W L T=L W R T=0.167 \\
L W N T=0.667
\end{gathered}
$$

\subsection{Network Connectivity Level}

The Network Connectivity Level (NCL) for each traffic flow (TF) and overall NCL are first calculated. The maximum number of nodes/vehicles directly connected to node/vehicle $i$ is referred to as the overall $\operatorname{NCL}(\alpha)$.

$$
\alpha_{i}(t)=\sum_{j} A(i, j, t)
$$

where $j$ is a potential connected neighboring vehicle.

The connectivity level (CL) $\beta$ for the TF of vehicle $i$ is calculated as

$$
\beta_{i}(t)=\sum_{j_{T F}} A\left(i, j_{T F}, t\right)
$$

where $j_{T F}$ is a vehicle sharing the TF with vehicle $i$.

On the same TF, the total CL for vehicle $i$ is given as;

$$
N C L_{i}(t)=\beta_{i}(t)+\left(\alpha_{i}(t) \times L W_{T F}\right)
$$

where LWTF is the lane weight for the lane occupied by vehicle $i$.

The normalized network connectivity level is given as;

$$
N C L_{\text {inorm }}=\frac{N C L_{i}}{\max \left(N C L_{x}\right)}
$$

\subsubsection{Average Distance Level}

The distance between the directly connected vehicles to vehicle $i$ is referred to as the overall average absolute distance, $\delta_{i}$ and is given as

$$
\delta_{i}=\frac{\sum_{j} \sqrt{\left(x_{j}-x_{i}\right)^{2}+\left(y_{j}-y_{i}\right)^{2}}}{N V}
$$

where $j$ is any vehicle connected to $i$, and $\mathrm{NV}$ is the total number of vehicles directly connected to $i$.

The average absolute distance, between vehicle $i$ and other vehicles in the same TF denoted as $\chi_{i}$ is calculated as;

$$
\chi_{i}=\frac{\sum_{j_{T F}} \sqrt{\left(x_{j}-x_{i}\right)^{2}+\left(y_{j}-y_{i}\right)^{2}}}{N V_{T F}}
$$

where $j$ is any vehicle connected directly to $i$ in the same $T F$, while, $N V_{T F}$ is the total number of vehicles in the same $T F$ directly connected to $i$.

The ADL for vehicle $i$ in $T F$ is given as

$$
\begin{aligned}
& A D L_{i}=\chi_{i}+\left(\delta_{i} \times L W_{T F}\right) \\
& A D L_{\text {inorm }}=\frac{A D L_{i}}{\max \left(A D L_{\chi}\right)}
\end{aligned}
$$

\subsubsection{Average Velocity Level}

By calculating the difference in the average velocities of all vehicles with that of the $\mathrm{CH}$ candidate, the overall Average Velocity Level (AVL), $\sigma_{\mathrm{i}}$, for the vehicles in a range is estimated as;

$$
\sigma_{i}=\frac{\sum_{N V}\left|V e l_{i}-V e l_{j}\right|}{N V}
$$

where, $V e l_{i}$ is the velocity of vehicle $i$.

The AVL, $\rho_{i}$, for vehicle $i$ and the $T F$ it belongs to determine as;

$$
\rho_{i}=\frac{\sum_{j T F}\left|V e l_{i}-V e l_{j T F}\right|}{N V_{T F}}
$$

where $j_{T F}$ is a vehicle in the same TF as vehicle $i$.

For vehicle $i$ in $T F$, the AVL is calculated as

$$
\begin{aligned}
& A V L_{i}=\rho_{i}+\left(\sigma_{i} \times L W_{T F}\right) \\
& A V L_{\text {inorm }}=\frac{A V L_{i}}{\max \left(A V L_{x}\right)}
\end{aligned}
$$

\subsection{CH Selection Algorithm}

Due to the mobility and the dynamic nature of nodes in VANET, the parameters of a node may change before the $\mathrm{CH}$ selection process is completed and the nodes that had highest probability for selection may all over sudden loose that status. To address this uncertainty, fuzzy logic is used in this study to introduce a rule based system with the 'IF-THEN' rules used in fuzzy inference system (FIS). As illustrated in Figure 3, the FIS membership functions (MFs) are defined under the fuzzifier while the rule base provides the fuzzy rules. The defuzzifier finally converts the fuzzy sets into output values which are crisp. 


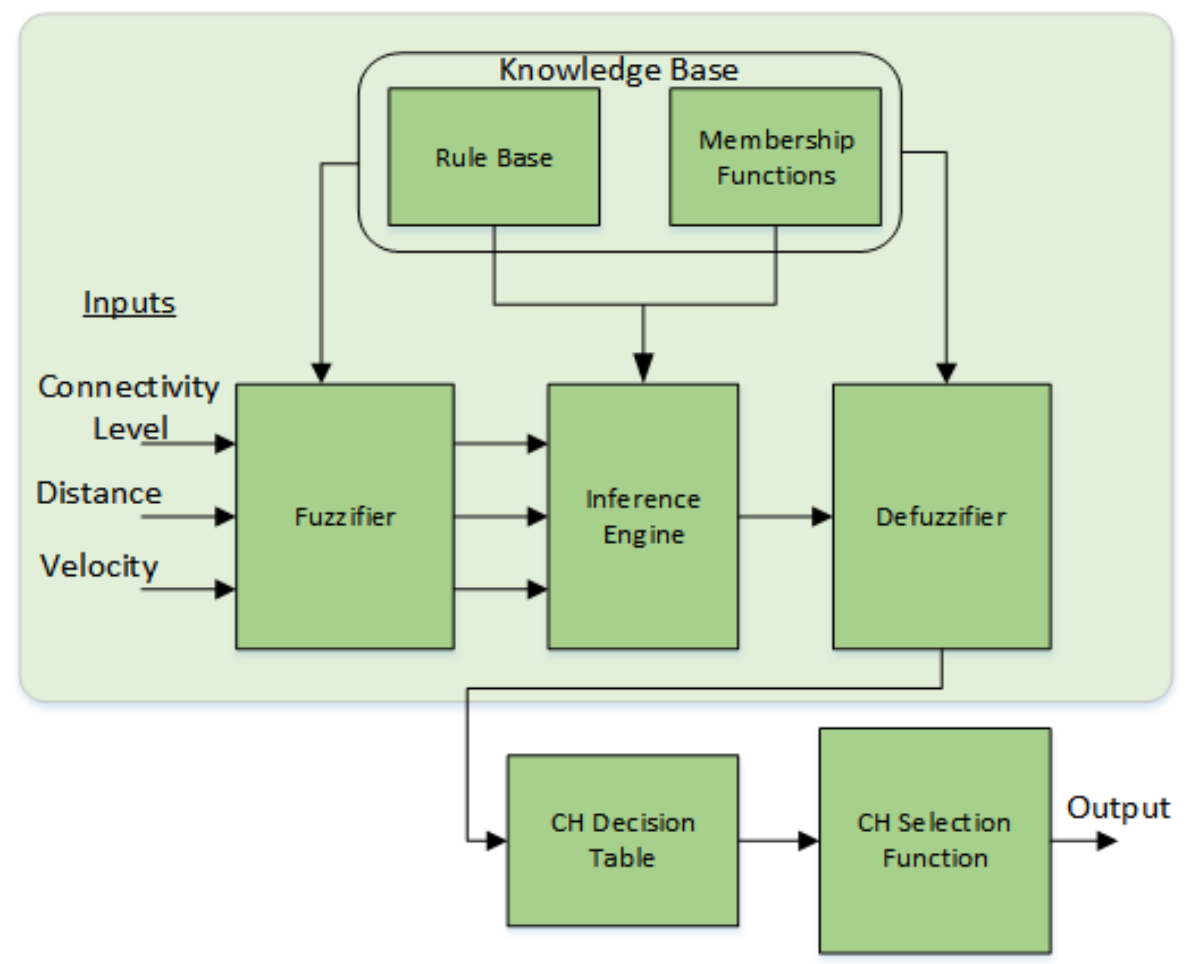

Figure 3. Fuzzy Logic CH Selection System

The results from equation 6, 10, and 14 for NCL, ADL, and AVL which vary from 0 to 1 are fed onto the fuzzifier of the fuzzy system where 5 MFs are specified for each as seen in Figure 4 - 6. The MFs of NCL are specified as Close (C), Very Closer (VC), Normal (N), Far (F), and Very Far (VF), while ADL has Near (N), Very Near (VN), Normal (N), Far (F), and Very Far (VF) MFs. Similarly, AVL has 5 MF as Slow (S), Very Slow (VS), Normal (N), Fast (F), and Very Fast (VF).

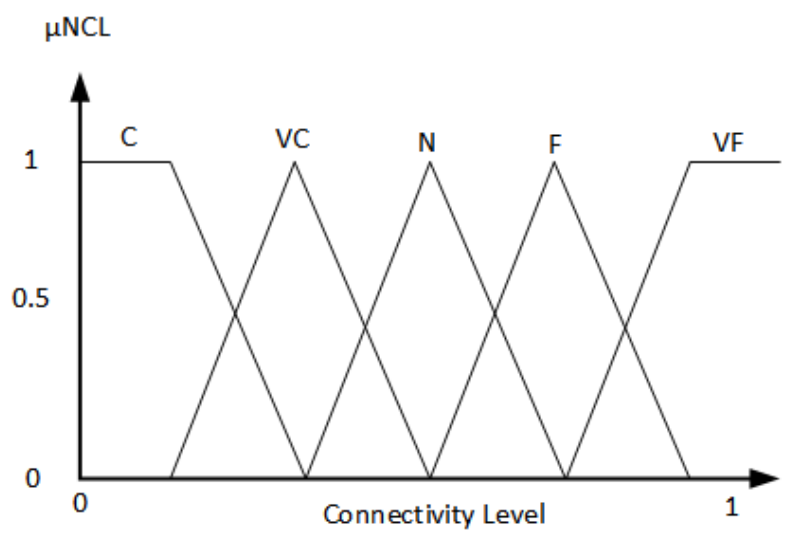

Figure 4. Normalized Network Connectivity Level

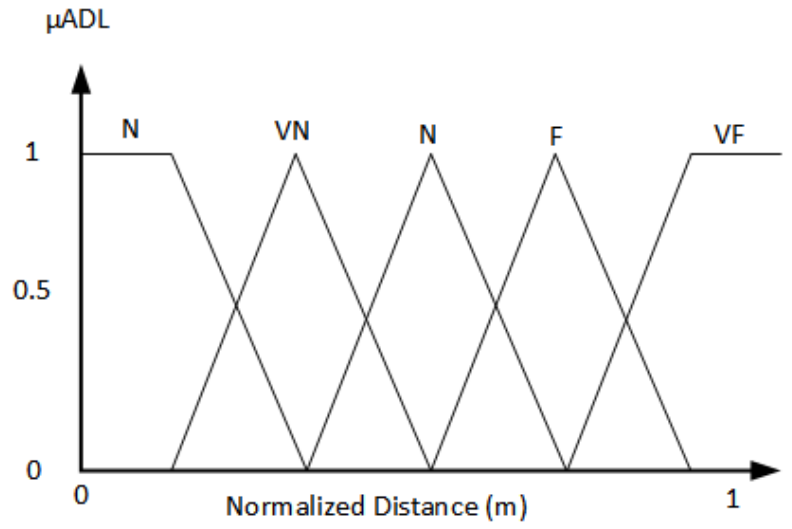

Figure 5. Normalized Average Distance Level

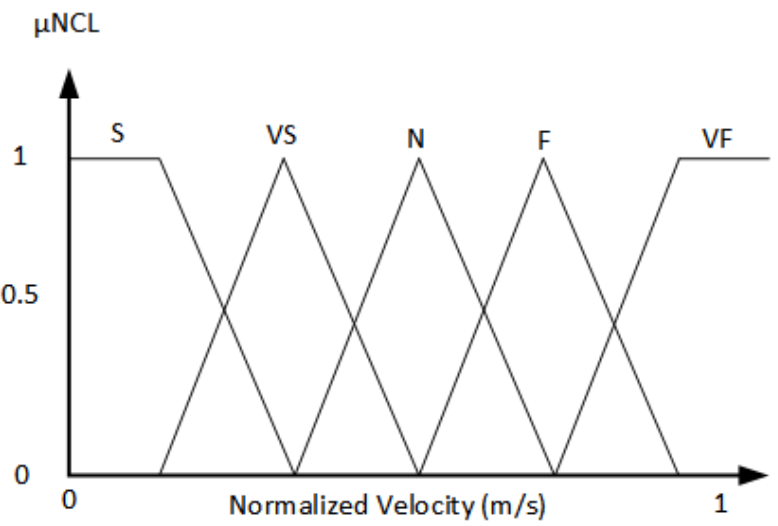

Figure 6. Normalized Average Velocity Level 
All vehicles compute and broadcast to their neighbors their Clusterhead Candidacy Level (CHL) weights along with their other parameters like velocity, position, etc. The highest weighted CHL vehicle is selected as the $\mathrm{CH}$ based on Eq. 15.

$$
\begin{gathered}
C H L_{i}=w_{1} * N C L_{\text {inorm }}(t)+w_{2} * A D L_{\text {inorm }}+ \\
A V L_{\text {inorm }}
\end{gathered}
$$

The vehicle with the highest CHL becomes the $\mathrm{CH}$.

\section{Simulation Results}

The VANET architecture proposed with a road network of three lane two-way highways was simulated on MATLAB with the performance metrics as the cluster size, $\mathrm{CH}$ duration, $\mathrm{CM}$ duration, and the average cluster lifetime among others. The road highway network used in this work is a two-way lane with length of $1500 \mathrm{~m}$ and a width of $24 \mathrm{~m}$ (12 $\mathrm{m}$ each direction) and has two RSUs each with a coverage radius of $1000 \mathrm{~m}$. Being a highway and assuming the random nature of vehicular speeds, the permitted maximum vehicle speed of $50 \mathrm{~km} / \mathrm{h}(13.89 \mathrm{~m} / \mathrm{s})$ is used in this work with each simulation being simulated $1000 s$ real time and averaged. Also, all the vehicles in the network are equipped with OBU and global positioning system (GPS) which allows the vehicle to know its position on the road segment. Using a QPSK modulation with a frequency of $5.9 \mathrm{GHz}$, the maximum communication range of a vehicle used is approximately $250 \mathrm{~m}$ radius. An RSU used is not limited to one way vehicles but can communicate with vehicle heading on different directions. With the grouping of vehicles dependent on it direction of movement, RSU plays a crucial role in vehicle synchronization. When a vehicle joins a road network, it broadcasts itself and waits for $5 s$ for communication from $\mathrm{CH}$ or RSU. If there is not communication from $\mathrm{CH}$ and a ping from 2 RSUs has been received, the vehicle aligns itself with an RSU with lower distance difference and ahead of it. After receiving communication with other vehicles within its range, a next phase of $\mathrm{CH}$ selection is initiated using fuzzy logics.

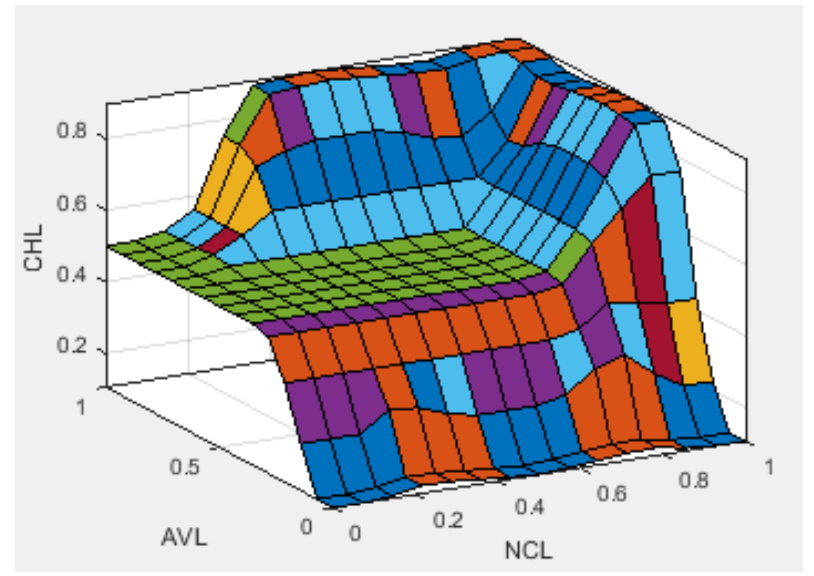

Figure 7. Surface Viewer: CHL vs AVL and NCL
Finally after $\mathrm{CH}$ selection, the $\mathrm{CH}$ sends a message to members informing them of their cluster parameters. It also sends a periodic invite messages to new vehicles seeking to join the cluster.

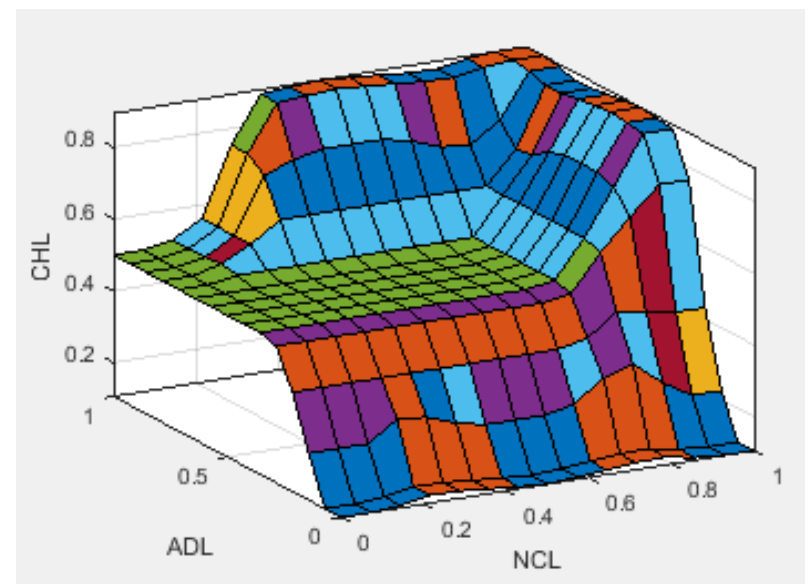

Figure 8. Surface Viewer: CHL vs ADL and NCL

Figure 7 and 8 show the surface view output of a fuzzy inference system. It can be seen that NCL is a crucial component in nomination of $\mathrm{CH}$. The output of a CHL system from both figures is directly proportional to the ADL, AVL and NCL.

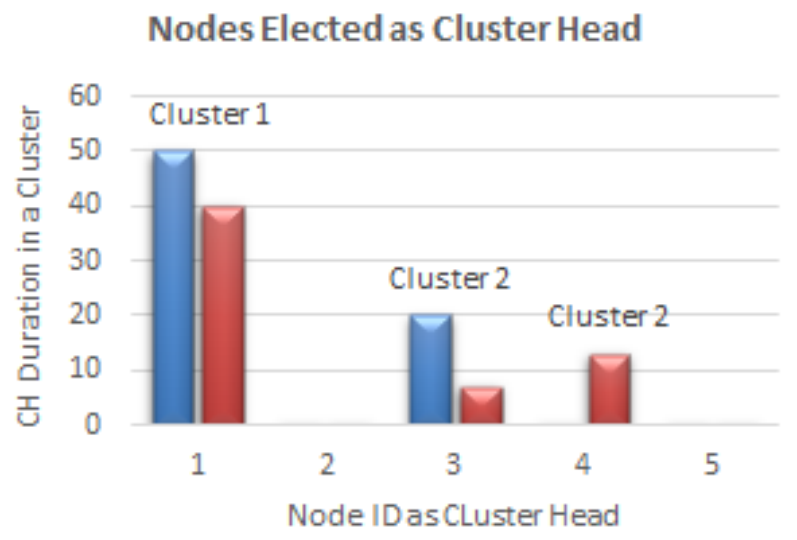

aWith LaneWeighting aWithout LaneWeighting

Figure 9. $\mathrm{CH}$ nodes for cluster 1 \& 2

To compare the effect of lane weighting on $\mathrm{CH}$ stability, a comparison of the same vehicular scenario with and without lane weighting was carried out. Vehicle with ID no. 1 is selected as the $\mathrm{CH}$ for cluster one while vehicle with ID no. 3 becomes a $\mathrm{CH}$ of cluster 2 both for two scenarios as seen in Figure 9. The duration of node 3 as $\mathrm{CH}$ without the use of lane weighting was shorter and node 4 was then elected as the new $\mathrm{CH}$ for the simulation period. Re-selection of $\mathrm{CH}$ is done when a $\mathrm{CH}$ leaves the cluster and another $\mathrm{CH}$ must be selected to take over the coordination operation of the cluster. Figure 10 shows the impact of various input parameters in CH selection. NCL 
is a crucial parameter in selection as in is important of a $\mathrm{CH}$ to have the highest number of directly connected vehicles as much as possible. Average distance level is the second most important parameter simply because a vehicle which is outside the cluster range or at the furthest point of the cluster cannot become a $\mathrm{CH}$, but rather the preference is for the node at the middle of the cluster as much as possible. Figure 11 indicates the average number of nodes in each cluster in VANET network.

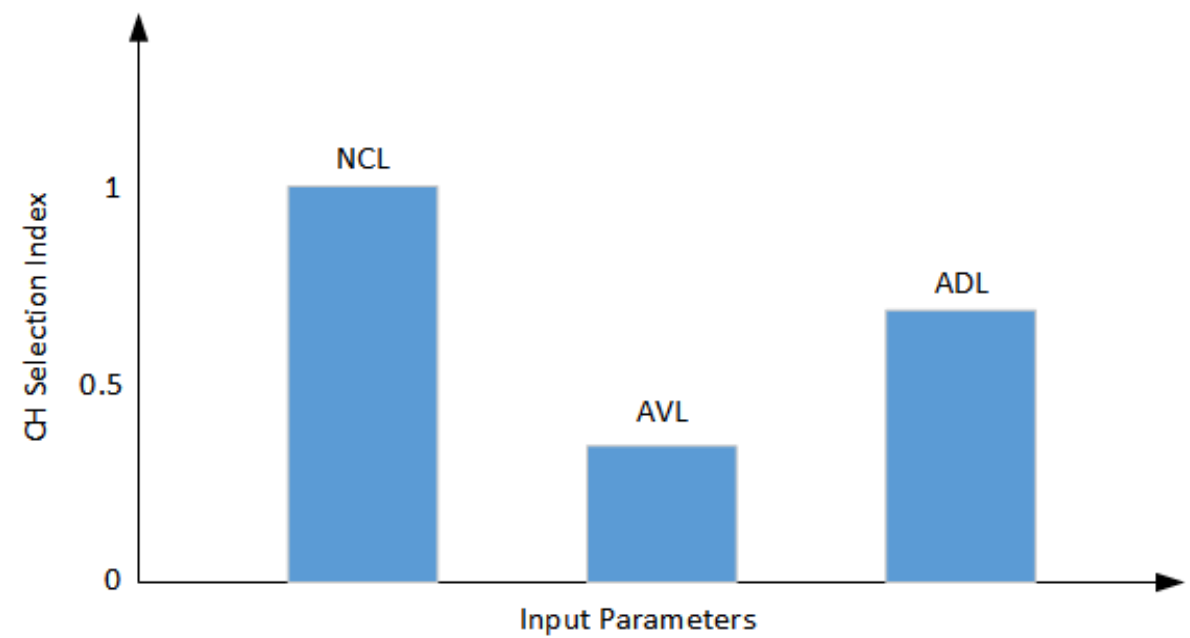

Figure 10. CH Selection Index based on Input Parameters

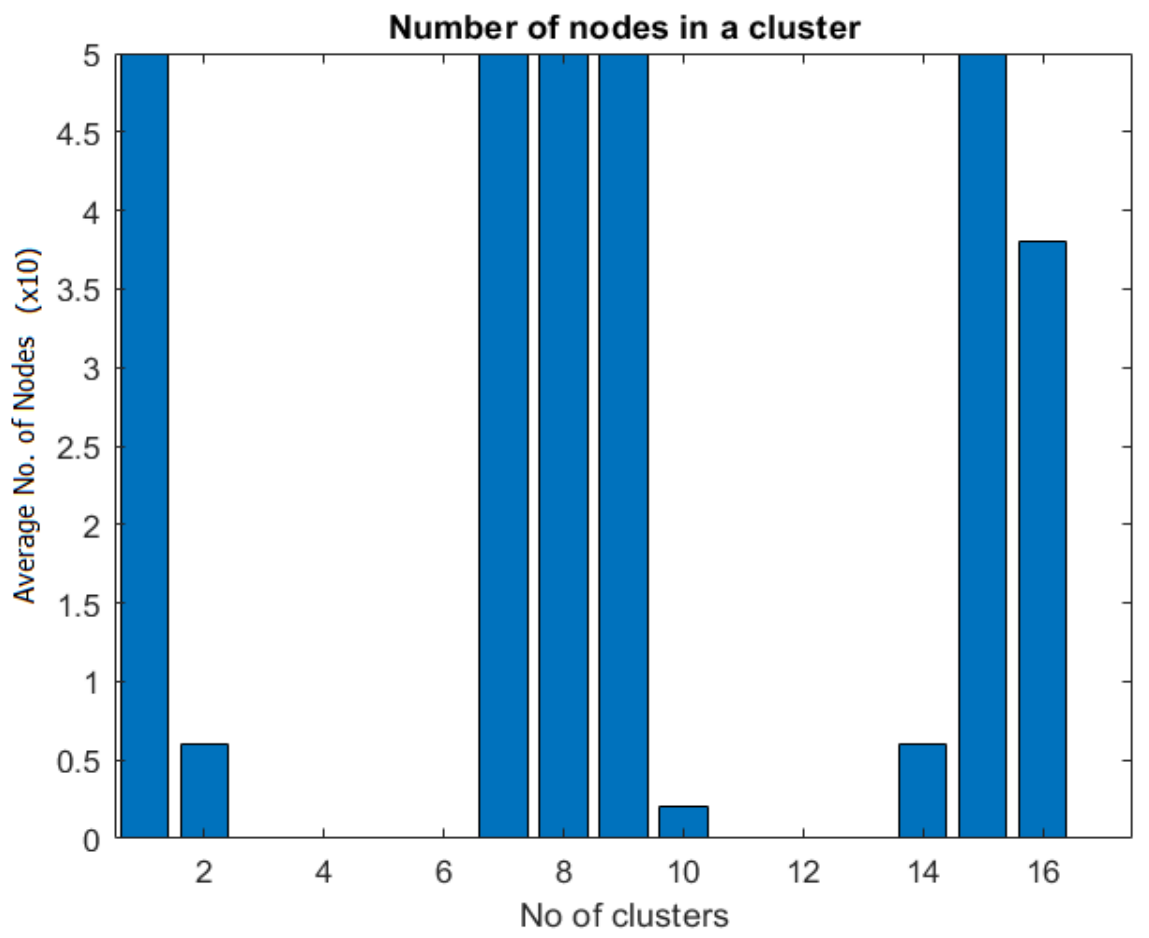

Figure 11. Average No. of Nodes in a Cluster 


\section{Conclusions}

An efficient fuzzy logic based clustering algorithm (SFCA) for VANET that reduces the reclustering overhead is proposed in this work. By using parameters like velocity, speed, and connectivity level of the vehicles, highly stable clusters that are ideal for VANETS can be achieved by implementing the proposed clustering scheme. It has also been shown that the lane weighting system can be effectively included in fuzzy logic based clustering systems for more effective and efficient clustering process.

\section{Limitation and Future Works}

This work was carried out using MATLAB simulation environment which has limited VANET features. This work can be replicated and outputs improved by running the simulation on specific vehicular designed environments. Also, further comparison can be done to validate the proposed system by comparing with the results generated by assuming the same velocity value for all vehicles. In future works, credit reward system can be incorporated to reward those nodes that effectively and efficiently participate in cluster data dissemination. These reward credits stored on a secure OBU can be used to acquired infotainment services and other services that are monetary like toll payments.

\section{REFERENCES}

[1] A. Abuashour and M. Kadoch, "Performance improvement of cluster-based routing protocol in VANET,” IEEE Access, vol. 5, pp. 15354-15371, 2017.

[2] P. K. Dharmawardena and Z. Wang, "Cluster Head selection Based Routing Protocol for VANET Using Bully Algorithm and Lamport Timestamp,” Int. J. Comput. Theory Eng., vol. 9, no. 3, pp. 218-222, 2017.

[3] S. Thirumurugan and J. B. Gnanadurai, "A Novel Application based Generic Cluster Creation Mechanism in Ad Hoc Networks,” Int. J. Comput. Networks Appl., vol. 4, no. 4, pp. 113-120, 2017.

[4] R. P. Surender Pal, "Vanet Based Simulation Using Cluster,” Int. J. Latest Res. Sci. Technol., vol. 6, no. 3, pp. 25-28, 2017.

[5] G. Martuscelli, A. Boukerche, L. Foschini, and P. Bellavista, "V2V protocols for traffic congestion discovery along routes of interest in VANETs: a quantitative study," Wirel. Commun. Mob. Comput., vol. 16, no. June 2009, pp. 2907 2923, 2016.

[6] C. Cooper, D. Franklin, M. Ros, F. Safaei, and M. Abolhasan, "A Comparative Survey of VANET Clustering Techniques,” IEEE Commun. Surv. Tutorials, vol. 19, no. 1, pp. 657-681, 2017.
[7] K. D. Singh, P. Rawat, and J. Bonnin, "Cognitive Radio for Vehicular Ad Hoc Networks ( CR-VANETs ): Approaches and Challenges," EURASIP J. Wirel. Commun. And Networking, vol. 1, no. 49, pp. 1-22, 2014.

[8] X. He, W. Shi, and T. Luo, "Survey of cognitive radio VANET,” KSII Trans. Internet Inf. Syst., vol. 8, no. 11, pp. 3837-3859, 2014.

[9] G. V. Rossi, Z. Fan, W. H. Chin, and K. K. Leung, "Stable clustering for Ad-Hoc vehicle networking," IEEE Wirel. Commun. Netw. Conf. WCNC, 2017.

[10] N. Nguyen-Thanh and I. Koo, "A Cluster-Based Selective Cooperative Spectrum Sensing Scheme in Cognitive Radio," Eurasip J. Wirel. Commun. Netw., vol. 2013, no. 1, pp. 19, 2013.

[11] A. H. Khalid, Z. Lian, L. Zaiyi, and M. Bobby, "Clustering and OFDMA-based MAC protocol (COMAC) for vehicular ad hoc networks,” EURASIP J. Wirel. Commun. Netw., vol. 2011, no. 1, p. 117, 2011.

[12] E. C. Eze, S. J. Zhang, E. J. Liu, and J. C. Eze, “Advances in Vehicular Ad-Hoc Networks (VANETs): Challenges and Road-Map for Future Development," Int. J. Autom. Comput., vol. 13, no. 1, pp. 1-18, 2016.

[13] M. Shoaib, W. C. Song, and K. H. Kim, “Cluster based data aggregation in vehicular adhoc network," Nets4Cars/Nets4 Trains, pp. 91-102, 2012.

[14] A. El Khatib, A. Mourad, H. Otrok, O. A. Wahab, and J. Bentahar, "A Cooperative Detection Model Based on Artificial Neural Network for VANET QoS-OLSR Protocol,” in 2015 IEEE International Conference on Ubiquitous Wireless Broadband, ICUWB 2015, 2015, pp. $1-5$.

[15] A. L. I. ÇALHAN, "A Fuzzy Logic Based Clustering Strategy for Improving Vehicular Ad-Hoc Network Performance," Sadhana - Acad. Proc. Eng. Sci., vol. 40, no. 2, pp. 351-367, 2015.

[16] R. S. Jadhav and M. M. Dongre, "Performance Enhancement of Vanets Using Fuzzy,” no. 2, pp. 39-42, 2017.

[17] B. Jinila, "Rough Set Based Fuzzy Scheme for Clustering and Cluster Head Selection in VANET," Elektron. IR Elektrotechnika, vol. 21, no. 1, pp. 54-59, 2015.

[18] P. Basu, N. Khan, and T. D. C. Little, “A Mobility Based Metric for Clustering in Mobile Ad Hoc," in 21st International Conference on Distributed Computing Systems Workshops, 2001, pp. 413-418.

[19] C. Shea, B. Hassanabadi, and S. Valaee, "Mobility-based clustering in VANETs using affinity propagation," in GLOBECOM - IEEE Global Telecommunications Conference, 2009, pp. 1-6.

[20] E. Souza, I. Nikolaidis, and P. Gburzynski, “A new Aggregate Local Mobility (ALM) clustering algorithm for VANETs," in IEEE International Conference on Communications, 2010, pp. 1-5.

[21] S. A. Mohammad and C. W. Michele, "Using traffic flow for cluster formation in vehicular ad-hoc networks," Proc. Conf. Local Comput. Networks, LCN, pp. 631-636, 2010. 
[22] J. P. Singh and R. S. Bali, "A hybrid backbone based clustering algorithm for vehicular ad-hoc networks," in International Conference on Information and Communication Technologies, 2015, vol. 46, pp. 10051013.

[23] A. W. Min, X. Zhang, and K. G. Shin, "Detection of small-scale primary users in cognitive radio networks," IEEE J. Sel. Areas Commun., vol. 29, no. 2, pp. 349-361, 2011.

[24] M. M. Caballeros Morales, C. S. Hong, and Y. C. Bang, "An Adaptable mobility-aware clustering algorithm in vehicular networks," in APNOMS 2011 - 13th Asia-Pacific Network Operations and Management Symposium: Managing Clouds, Smart Networks and Services, Final Program, 2011, pp. 1-6.

[25] M. Mottahedi, S. Jabbehdari, and S. Adabi, "IBCAV: Intelligent Based Clustering Algorithm in VANET," IJCSI Int. J. Comput. Sci. Issues, vol. 10, no. 1, pp. 538-543, 2013.

[26] K. A. Hafeez, L. Zhao, Z. Liao, and B. N. W. Ma, “A fuzzy-logic-based cluster head selection algorithm in VANETs," in IEEE International Conference on Communications, 2012, pp. 203-207. 